\title{
Roedores y murciélagos en espacios verdes en San Cristóbal de Las Casas, Chiapas, México
}

\author{
Antonio García-Méndez ${ }^{1}$, Consuelo Lorenzo ${ }^{1 *}$, \\ Luis-Bernardo Vazquez ${ }^{1}$ y Rafael Reyna-Hurtado ${ }^{2}$
}

Introduction: The demands of space for housing, industrial, commercial, and recreation space, are increasing as the human population continues to focus on urban areas. This implies an increase and constant degradation of natural environments. Despite the negative effect that the urban areas have on the biodiversity, these can be considered as important areas for the conservation of native flora and fauna. This is because urban areas have different types of land uses, where green spaces, represent an important component for the conservation of biological diversity. This study estimated the richness and abundance of rodents and bats in urban and non-urban green spaces in the city of San Cristobal de Las Casas (SCLC), Chiapas, Mexico. Given the great variety of types of green space in an urban area, the richness and abundance of rodents and bats in these areas will depend on the characteristics of the vegetation (richness and abundance of vegetal species) and the formation of the surrounding matrix (types of uses of soil around the green space).

Methods: This study was conducted in 10 sites with presence of green spaces in the urban area and the periphery of SCLC. The rodents and bats were captured monthly from March to August 2013, for three consecutive nights per site. We compared the richness of species, relative abundance and diversity index between urban and non-urban green spaces.

Results: There were a total of 12 species and 108 individuals, belonging to three families, Muridae, Phyllostomidae, and Vespertilionidae. In the non-urban green spaces were captured 55 individuals of four species of native rodents, and 19 individuals of three species of bats. In the urban green spaces were captured 11 individuals of three species of rodents (a species was native), and 23 individuals of four species of bats. For the richness of species of mammals, the correlation analysis showed very low values of relationship regarding to the independent variables.

Discussion and conclusions: The number of species recorded in this study in the two groups of green spaces (urban and non-urban), show that these spaces can hold more than $40 \%$ of the total number of species recorded for the municipality of San Cristóbal de Las Casas. The capture of larger number of individuals of species of native rodents in the non-urban green spaces may be that in these areas there is greater availability of resources such as food, shelter and breeding sites, since these sites are part of a remnant of forest. In the case of bats, there were not significant differences in their diversity between urban and non-urban green spaces. This study found that the urban and non-urban green spaces in the interior and periphery of the city of SCLC, are important for the native fauna of rodents and bats. In this way, preserving green areas on the periphery of an urban landscape is decisive for the conservation of the native fauna of the region, since they are reservoirs of their diversity.

Key words: bats, biodiversity, conservation, green spaces, mammals, rodents, urban areas.

${ }^{1}$ El Colegio de La Frontera Sur, Unidad San Cristóbal. Carretera Panamericana y Periférico Sur s/n, Barrio de María Auxiliadora. San Cristóbal de Las Casas, Chiapas, 29290, México. E-mail: antgarcia@ecosur.edu.mx (AGM), clorenzo@ ecosur.mx (CL), lbvazquez@ecosur.mx (LBVH).

${ }^{2}$ El Colegio de la Frontera Sur, Unidad Campeche. Av. Rancho Polígono 2-A, Colonia Ciudad Industrial. Lerma Campeche, Campeche, 24500, México. E-mail: rreyna@ecosur.mx (RRH).

"Corresponding author 
A medida que la población humana continúa concentrándose en las zonas urbanas, se incrementan las demandas de espacios para viviendas, espacio industrial, comercial y de recreación, lo cual implica un deterioro constante en el ambiente y un aumento de los espacios verdes. En general, los espacios verdes en las zonas urbanas pueden servir como áreas de conservación de la flora y fauna nativa. Se estimó la riqueza y abundancia de roedores y murciélagos en espacios verdes urbanos y no urbanos, en la ciudad de San Cristóbal de Las Casas (SCLC), Chiapas, México. Se capturaron roedores y murciélagos en 10 espacios verdes distribuidos en el interior y periferia de la ciudad, los cuales se agruparon en urbanos y no urbanos. Se registraron un total de 108 individuos correspondientes a 12 especies pertenecientes a tres familias, Muridae, Phyllostomidae y Vespertilionidae. En los espacios verdes no urbanos se capturaron 55 individuos de cuatro especies de roedores nativos y 19 individuos pertenecientes a tres especies de murciélagos. En los espacios verdes urbanos, se capturaron 11 individuos de tres especies de roedores (una especie fue nativa) y 23 individuos de cuatro especies de murciélagos. Se encontró que los espacios verdes urbanos y no urbanos de SCLC son importantes para la fauna nativa de roedores y murciélagos, al albergar el $40 \%$ de su fauna reportada. El conservar áreas verdes en la periferia de un paisaje urbano es determinante para la conservación de la fauna nativa de la región, debido a que son reservorios de diversidad.

Palabras clave: áreas urbanas, biodiversidad, conservación, espacios verdes, mamíferos, murciélagos, roedores.

A medida que la población humana continúa concentrándose en las zonas urbanas, se incrementan las demandas de espacios para viviendas, espacio industrial, comercial y de recreación, lo cual implica un aumento del deterioro constante de los ambientes naturales (Vitousek et al. 1997; McKinney 2002; Miller y Hobbs 2002; Forman 2008).

A pesar de las repercusiones y el efecto negativo que tienen las zonas urbanas sobre la diversidad biológica, éstas pueden considerarse como áreas importantes para la conservación de la flora y fauna nativa (McKinney 2008). Lo anterior se debe a que las zonas urbanas son sistemas heterogéneos, formados por diferentes tipos de usos de suelo, donde los espacios verdes en su interior representan un componente importante para la conservación de la diversidad biológica, que representa una opción de hábitat para la flora y fauna nativa (Sukopp y Werner 1982; McDonnell y Pickett 1990, 1993; González-García y Gómez-Sal 2008; Alberti 2009).

Los espacios verdes son áreas que presentan un porcentaje mayor de cobertura de algún tipo de vegetación (nativa o inducida) y una baja cobertura de estructuras urbanas (edificios, casas, comercios, plazas; Forman 2008). Existen una gran variedad de espacios verdes en las zonas urbanas, por ejemplo: áreas de juegos, campos deportivos, humedales, reservas naturales públicas y particulares, huertas familiares, parques urbanos y periurbanos, vegetación ribereña y jardines públicos y particulares (McKinney 2002; Forman 2008; Wu 2008; Godbard et al. 2009; González-García et al. 
2009). De esta manera, algunos espacios verdes pueden ser reservorios de la riqueza de especies de fauna y flora, al fungir como corredores naturales y ser usados como vías de desplazamiento por la fauna (Mason et al. 2007; Forman 2008; Loram et al. 2011).

No obstante, no todos los espacios verdes urbanos son importantes para la conservación de la flora y fauna nativa. La importancia de los espacios verdes como hábitats para la fauna silvestre depende principalmente del tipo y estructura de la vegetación, así como de la matriz en la que se encuentran inmersos (Mahan y O'Connell 2005; Smith et al. 2006). De esta manera, se ha encontrado que los espacios verdes que presentan mayor área con vegetación nativa o vegetación con elementos nativos e inmersos en una matriz con poco disturbio humano, albergan mayor riqueza de especies de fauna (Sauvajot et al. 1998; Mason et al. 2007).

San Cristóbal de Las Casas (SCLC), Chiapas, es una de las ciudades de mayor crecimiento en el país en las últimas décadas, con cerca de 160,000 habitantes y con un promedio anual de crecimiento del 3.3\% (INEGI 2010). Al mismo tiempo, la población humana de esta ciudad en crecimiento ha ocupado áreas agrícolas y espacios verdes de la periferia. Sin embargo, SCLC aún presenta y conserva espacios verdes en el interior de la ciudad y en la periferia (huertas familiares, áreas de cultivo, jardines particulares, parques urbanos, manantiales y humedales de montaña; Aubry 2008; Vásquez 2010) que podrían ser importantes para la conservación de la diversidad biológica. Por lo anterior, en este estudio se investigaron diferentes espacios verdes no urbanos (en la periferia) y urbanos (en el interior) en la ciudad de San Cristóbal de Las Casas, para describir la manera en que son utilizados por mamíferos nativos con diferentes requerimientos ecológicos, como son los roedores y los murciélagos.

Considerando que la diversidad local de especies de mamíferos nativos en áreas urbanas es dependiente del contexto espacial a nivel de paisaje y de la cercanía con las áreas naturales, nos planteamos como objetivo describir la riqueza, diversidad y abundancia de estos grupos de mamíferos en los espacios verdes interiores y periféricos así como en sitios con diferente uso de suelo alrededor de los mismos. Los resultados se relacionaron con la abundancia y riqueza de especies de árboles en los espacios verdes con el fin de determinar su influencia en la diversidad de roedores y murciélagos.

\section{Material}

\section{y Métodos}

El estudio se llevó a cabo en 10 sitios con presencia de espacios verdes en la zona urbana y periferia de la ciudad de SCLC, Chiapas, México (Fig. 1), de diversos tipos como: parque urbano, humedal, jardín particular, huerta familiar, manantial, áreas de pastoreo y áreas conservadas. Los límites urbanos de la ciudad considerados en este estudio, son los establecidos por INEGI (2012). Los sitios de muestreo se ubican en la región Altos de Chiapas a una altitud de 2,120 msnm, en un valle rodeado por áreas montañosas que superan los 2,700 msnm en sus puntos más altos (Mera-Ovando 1984; García-García 2005). El clima es templado sub-húmedo con lluvias en verano, con una precipitación anual de 1,000 a 2,500 mm y una temperatura media anual de $14.4{ }^{\circ} \mathrm{C}$ con algunas heladas invernales ocasionales (INEGI 2012; CEIEG 2012).

Selección de los espacios verdes (sitios de muestreo). Se analizó una imagen de satélite del 2012 de la ciudad de San Cristóbal de Las Casas, en ella se trazaron polígonos $\geq$ 
$1,000 \mathrm{~m}^{2}$, con el fin de identificar y obtener el número y tipo de los diferentes usos de suelo. Este análisis se realizó con la ayuda del programa Google Earth Pro. Los tipos de usos de suelo considerados en este estudio fueron: 1) los espacios verdes, aquéllos que presentaron cobertura arbórea, remanentes de bosque, vegetación secundaria, vegetación ribereña y jardines públicos y particulares; 2) los espacios abiertos, terrenos baldíos sin cobertura vegetal y sin construcciones (urbanización), áreas deportivas y minas abiertas; 3) las áreas de cultivo, espacios con cultivos de hortalizas y maíz; 4) las zonas de pastoreo, sitios donde hubo presencia de ganado vacuno y ovino; 5) los cuerpos de agua, lagos, lagunas y manantiales; y 6) la cobertura urbana, superficies impermeables (cubiertas por cemento, asfalto o cualquier material no permeable), centros comerciales, edificios, casas y construcciones realizadas por el hombre.

Posteriormente se procedió a la revisión y exploración en campo, en donde se consideraron varios aspectos para seleccionar los sitios de muestreos, como la accesibilidad al lugar (público o particulares), y seguridad tanto para el personal de trabajo como por el equipo de muestreo. De esta manera, se seleccionaron 10 espacios verdes (sitios de muestreos) tomando en cuenta, el tamaño (área), ubicación (urbano o no urbano), y tipo de espacio verde (parque, jardín, área conservada).

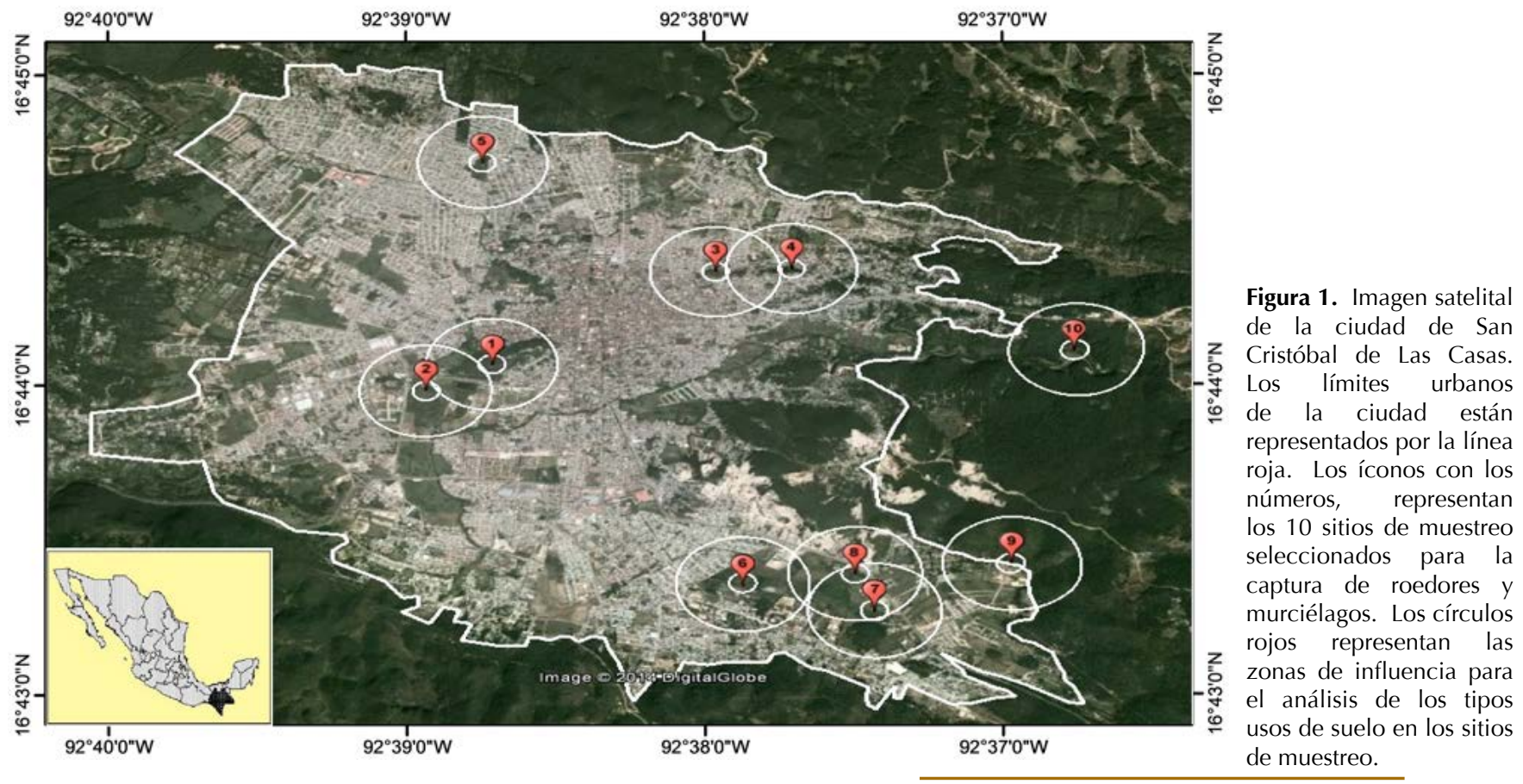

Los espacios verdes seleccionados para el muestreo se clasificaron en dos grandes grupos: 1) según su ubicación, en espacios verdes urbanos (en el interior de SCLC, delimitado por el circuito Periférico) y espacios verdes no urbanos (fuera de los límites urbanos de la ciudad) y 2) según el tipo, de acuerdo al porcentaje de zonas urbanas y de otros tipos de usos de suelo alrededor de los espacios verdes. De esta manera, dos sitios, La UNICH y El Encuentro fueron espacios verdes no urbanos, mientras que los ocho restantes se agruparon como espacios verdes urbanos (Tabla 1). Para conocer el número de los diferentes usos de suelo alrededor de los espacios verdes, se trazaron zonas de influencia (buffers) de $500 \mathrm{~m}$ de radio desde el centro de los espacios verdes 
muestreados (Fig. 1), porque es posible que los usos de suelo que rodean el espacio verde influyan en la presencia y captura de murciélagos y roedores. Se tomó en cuenta que los roedores presentan ámbitos hogareños de hasta $500 \mathrm{~m}$ de radio (Contreras y Rosi 1981; García-Estrada et al. 2002; Corbalán y Ojeda 2005); mientras que, los murciélagos tienen requerimientos biológicos diferentes, los cuales pueden ser cubiertos en distintos ámbitos hogareños, para lo cual consideramos la posibilidad de registrar la actividad de murciélagos en esta zona de influencia (Fleming 1981; Pearson et al. 1987; Gorresen et al. 2005; Klingbeil y Willig 2009). En cada zona de influencia, se delinearon polígonos para conocer el área (en hectáreas) de los diferentes usos de suelo (espacios abiertos, áreas de cultivo, zonas de pastoreo, cuerpos de agua y coberturas urbanas) presentes alrededor de los espacios verdes, a través del software Google Earth Pro.

\begin{tabular}{|c|c|c|c|c|c|c|c|c|c|}
\hline \multirow{6}{*}{$\begin{array}{l}\text { Tabla 1. Características } \\
\text { de } 10 \text { espacios verdes } \\
\text { muestreados en San } \\
\text { Cristóbal de Las Casas, } \\
\text { Chiapas, México. Se } \\
\text { incluyen la riqueza y } \\
\text { abundancia local de } \\
\text { árboles, así como el } \\
\text { área (ha) ocupada por } \\
\text { los diferentes usos de } \\
\text { suelo en las zonas de } \\
\text { influencia (500 m a } \\
\text { la redonda). } \mathrm{N}=\text { sitio } 3 \\
\text { urbano; } \mathrm{NU}=\text { sitio no } \\
\text { urbano. }\end{array}$} & \multirow{2}{*}{\multicolumn{2}{|c|}{ No. Sitios }} & \multirow[b]{2}{*}{$\begin{array}{l}\text { Tipo de espacio } \\
\text { verde }\end{array}$} & \multicolumn{2}{|c|}{ Atributos locales } & \multicolumn{4}{|c|}{$\begin{array}{l}\text { Área (ha) ocupada por distintos usos de suelo en } \\
\text { las zonas de influencia de } 500 \mathrm{~m} \text { de radio }\end{array}$} \\
\hline & & & & $\begin{array}{l}\text { Riqueza } \\
\text { de } \\
\text { árboles }\end{array}$ & $\begin{array}{c}\text { Número } \\
\text { de } \\
\text { árboles/ } \\
\text { parcela }\end{array}$ & $\begin{array}{l}\text { Espacios } \\
\text { abiertos }\end{array}$ & $\begin{array}{c}\text { Áreas } \\
\text { urbanizadas }\end{array}$ & $\begin{array}{l}\text { Cuerpos } \\
\text { de agua }\end{array}$ & $\begin{array}{c}\text { Zonas } \\
\text { de pastoreo }\end{array}$ \\
\hline & & El Cerrito (N) & Parque urbano & 12 & 145 & 7.65 & 41.42 & 2.12 & 0.64 \\
\hline & & La Kisst (N) & Humedal & 8 & 30 & 27.18 & 20.86 & 1.59 & 3.43 \\
\hline & & Na-Bolom (N) & Jardín particular & 10 & 52 & 0 & 67.76 & 0 & 0 \\
\hline & 4 & Saldivar (N) & Huerta familiar & 9 & 27 & 3.26 & 57.00 & 0 & 0 \\
\hline & 5 & La Hormiga (N) & Manantial & 4 & 19 & 2.35 & 65.30 & 0.53 & 0 \\
\hline & 6 & La Albarrada* (N) & $\begin{array}{l}\text { Cultivos y zonas } \\
\text { de pastoreo }\end{array}$ & 3 & 52 & 10.96 & 26.30 & 0.48 & 1.50 \\
\hline & 7 & Los Humedales* $(\mathrm{N})$ & Humedal & 4 & 25 & 16.93 & 15.59 & 0.74 & 1.19 \\
\hline & 8 & Navajuelos (N) & Manantial & 2 & 26 & 16.92 & 15.72 & 0.74 & 2.01 \\
\hline & 9 & La UNICH (NU) & $\begin{array}{l}\text { Área } \\
\text { conservada }\end{array}$ & 8 & 66 & 18.22 & 6.17 & 0 & 0 \\
\hline *Únicos sitios con & 10 & El Encuentro (NU) & $\begin{array}{l}\text { Área } \\
\text { conservada }\end{array}$ & 8 & 78 & 4.08 & 0 & 1.54 & 0 \\
\hline
\end{tabular}

Por otro lado, en los sitios seleccionados para los muestreos de roedores y murciélagos, se hizo un análisis local de la vegetación, que consistió en trazar parcelas de 1,000 m² en los sitios de muestreo. El número de parcelas trazadas en los sitios varió de uno a seis, según el tamaño de los espacios verdes. En cada parcela se anotó la riqueza y abundancia de especies de árboles. Para la identificación de las especies de árboles se consultaron las referencias bibliográficas sobre la flora de San Cristóbal de Las Casas (Galindo-Jaimes et al. 2008; Ramírez-Marcial et al. 2010), además se realizaron colectas de cada especie para su corroboración e identificación en el herbario de El Colegio de La Frontera Sur (ECOSUR-SCLC). En algunos sitios ya existían con anterioridad inventarios de la vegetación, y fue posible acceder a la base de datos correspondiente (RamírezMarcial 2013 com. per.). 
Muestreo de mamíferos pequeños. Captura de roedores. Los roedores se capturaron mensualmente de marzo a agosto de 2013, por tres noches consecutivas por sitio. Se utilizaron 100 trampas Sherman, cebadas con avena, semillas de girasol y extracto de vainilla para aumentar la probabilidad de captura de los roedores. Las trampas se colocaron a partir de las 17:00 hrs en transectos lineales abarcando la mayor parte del área de los sitios muestreados (espacios verdes seleccionados) y se revisaron al día siguiente. Se registró la geoposición de cada captura y los individuos se marcaron con pintura de esmalte negro (marca temporal) en las uñas de los dedos, como una modificación al marcaje tradicional de ectomización de falanges (marca permanente; Romero-Almaraz et al. 2007). Los individuos capturados se identificaron en campo hasta nivel de especie con la ayuda de referencias bibliográficas especializadas, tales como la clave de Álvarez (1959) y el libro ilustrado de Reid (1997).

Captura de murciélagos. Los murciélagos se capturaron mensualmente de marzo a agosto de 2013, por tres noches consecutivas por sitio, utilizando tres redes de niebla de $12 \mathrm{~m}$ de longitud. Las redes de niebla se colocaron en un trayecto lineal con una separación de 100 m entre cada red y permanecieron abiertas durante seis horas, de las 18:00 a las 24:00 hrs. Los individuos capturados fueron identificados a nivel de especie siguiendo la clave de identificación de Medellín et al. (1997) y el libro ilustrado de Reid (1997), y se liberaron en el lugar de captura. Se registró la geoposición de la captura, y a los individuos se les colocaron marcas semi-permanentes en las membranas dactilares del ala derecha mediante pequeñas perforaciones, tomando en cuenta una matriz de combinaciones, con una aguja (de $0.5 \mathrm{~mm}$ de diámetro) esterilizada y posteriormente se desinfectó con peróxido de hidrógeno como antiséptico (Kunz 1996).

Análisis estadísticos. Estimamos el índice de diversidad de Shannon-Wiener $\left(\mathrm{H}^{\prime}\right)$, la riqueza de especies y la abundancia relativa de roedores y murciélagos (separadamente y en conjunto) para cada uno de los espacios verdes. Utilizamos pruebas de $t$ de Student para determinar si existen diferencias significativas en estos parámetros entre los espacios verdes urbanos y no urbanos. Los análisis se llevaron a cabo en el programa Past 3.0 (Hammer et al. 2001). Por otro lado, con el fin de conocer la relación de las variables locales y del paisaje con la riqueza y la abundancia de especies de roedores y murciélagos, realizamos análisis de correlación simple de Pearson, para el total de los animales capturados.

El tamaño y estructura en hectáreas de los diferentes usos de suelo registrados en los espacios verdes con las zonas de influencia para el análisis de las características de los sitios seleccionados, se muestran en la Tabla 1. Un espacio verde urbano (El Encuentro) presentó la mayor riqueza y abundancia de árboles, mientras que en los espacios verdes no urbanos se registraron menor número de especies y abundancias (Tabla 2).

Se registraron un total de 12 especies y 108 individuos de mamíferos, pertenecientes a las familias Muridae, Phyllostomidae y Vespertilionidae. El orden Rodentia fue el más diverso con seis especies y 66 individuos, y Peromyscus levipes fue la especie más 
abundante con 42 individuos capturados durante todo el estudio. En contraste, del orden Chiroptera se capturaron seis especies y 42 individuos, y la especie más abundante fue Artibeus lituratus con 18 individuos capturados.

\begin{abstract}
Tabla 2. Riqueza y abundancia de los árboles registrados en los 10 espacios verdes muestreados en San Cristóbal de Las Casas, Chiapas, México.
\end{abstract}

Los números representa los sitios muestreados: 1. El Cerrito, 2. La Kisst, 3. NaBolom, 4. Saldivar, 5. La Hormiga, 6. La Albarrada, 7. Los Humedales, 8. Navajuelos, 9. La UNICH, 10. El Encuentro.

\begin{tabular}{|c|c|c|c|c|c|c|c|c|c|c|}
\hline Especies/ Número del sitio & 1 & 2 & 3 & 4 & 5 & 6 & 7 & 8 & 9 & 10 \\
\hline Cupressus lusitanica & 50 & & 15 & 4 & 7 & 39 & & 20 & 16 & 20 \\
\hline Crataegus mexicana & 37 & & & 4 & & & & & 15 & 5 \\
\hline Fraxinus uhdei & 7 & 6 & & & 4 & & & & 10 & 16 \\
\hline Calliandra grandiflora & 3 & & & & & & & & 2 & 23 \\
\hline Pinus pseudostronsis & 15 & & & & & & & & 7 & 4 \\
\hline Populus tremuloides & & & & & & 12 & 10 & & & \\
\hline Cornus excelsa & 13 & 2 & & & & & & & & 3 \\
\hline Ligustrum japonicum & 3 & & & & & & & & 7 & 4 \\
\hline Eriobotrya japonica & & & 6 & 1 & & & & & 5 & \\
\hline Crataegus pubescens & & & 2 & & & & 4 & 6 & & \\
\hline Garrya laurifolia & 8 & & & & & & & & 4 & \\
\hline Prunus persica & 6 & & & 1 & 4 & & & & & \\
\hline Pinus tecunumanii & & & 10 & & & & & & & \\
\hline Datura candida & & & 9 & & & & & & & \\
\hline Taxodium mucronatum & & 5 & & & 4 & & & & & \\
\hline Verbesina perymenioides & & & & & & & 9 & & & \\
\hline Pyrus malus & & & 1 & 5 & & & & & & \\
\hline Alnus acuminata & & 4 & & & & & & & & \\
\hline Rhamnus capreifolia & & & 3 & & & 1 & & & & \\
\hline Quercus peduncularis & & 4 & & & & & & & & \\
\hline Acer negundo & & 4 & & & & & & & & \\
\hline Pinus oocarpa & & 4 & & & & & & & & \\
\hline Malus sp. & & & & 3 & & & & & & \\
\hline Abies guatemalensis & & & & 3 & & & & & & \\
\hline Ficus sp. & & & & 3 & & & & & & \\
\hline Cydonia oblonga & & & & 3 & & & & & & \\
\hline Psidium guajava & & & & & & & & & & 3 \\
\hline Prunus serotina & 1 & 1 & & & & & & & & \\
\hline Eleocharis filiculmis & & & & & & & 2 & & & \\
\hline Randia aculeata & & & 2 & & & & & & & \\
\hline Prunus sp. & & & 1 & & & & & & & \\
\hline Eucalyptus sp. & 1 & & & & & & & & & \\
\hline Casimiroa edulis & 1 & & & & & & & & & \\
\hline Total & 145 & 30 & 52 & 27 & 19 & 52 & 25 & 26 & 66 & 78 \\
\hline
\end{tabular}

En el sitio 1, El Cerrito (parque urbano), se registraron tres especies, el roedor Reithrodontomys mexicanus fue la especie más abundante y seis individuos de dos especies de quirópteros. En el sitio 2, La Kisst (humedal), se capturó un individuo de 
R. mexicanus. En el sitio 3, Na-Bolom (jardín particular), se capturaron cinco especies, un roedor (Rattus rattus) y cuatro murciélagos, donde $A$. lituratus fue la especie más abundante con tres individuos. Para el sitio 5, La Hormiga (manantial), se registraron dos especies, un ratón y un murciélago, con $A$. lituratus como la especie más abundante con seis individuos. En el sitio 6, La Albarrada (cultivos y pastoreo), se capturaron tres especies, un roedor y dos quirópteros, y A. lituratus fue la especie más abundante con tres individuos. Para el sitio 9, La UNICH (área conservada), se registraron ocho especies, seis roedores y dos murciélagos, y Dermanura azteca fue la especie más abundante con 17 individuos. Finalmente en el sitio 10, El Encuentro (área conservada), se capturaron cuatro especies, tres roedores y un murciélago, y $P$. levipes fue la especie más abundante con 41 individuos. En tres sitios, el 4 Saldívar (huerta familiar), el 7 Los Humedales (humedal) y el 8 Navajuelos (manantial), no se colectaron ni ratones ni murciélagos (Tabla 3).

\begin{tabular}{|c|c|c|c|c|c|c|c|c|c|c|c|c|}
\hline Orden & Especies/Número del sitio & 1 & 2 & 3 & 4 & 5 & 6 & 7 & 8 & 9 & 10 & Total \\
\hline \multirow[t]{6}{*}{ Rodentia } & Peromyscus levipes & & & & & & & & & 1 & 41 & 42 \\
\hline & Peromyscus mexicanus & & & & & & & & & 7 & 1 & 8 \\
\hline & Reithrodontomys mexicanus & 6 & 1 & & & & & & & 1 & 2 & 10 \\
\hline & Sigmodon hispidus & & & & & & & & & 2 & & 2 \\
\hline & Mus musculus* & & & & & 1 & & & & & & 1 \\
\hline & Rattus rattus* & & & 1 & & & 2 & & & & & 3 \\
\hline \multirow[t]{7}{*}{ Chiroptera } & Artibeus lituratus & 5 & & 3 & & 6 & 3 & & & 1 & & 18 \\
\hline & Dermanura azteca & & & & & & & & & 17 & & 17 \\
\hline & Lasiurus intermedius & & & 1 & & & & & & & & 1 \\
\hline & Lasiurus cinereus & & & & & & & & & & 1 & 1 \\
\hline & Eptesicus fuscus & 1 & & 2 & & & 1 & & & & & 4 \\
\hline & Eptesicus brasiliensis & & & 1 & & & & & & & & 1 \\
\hline & Total & 14 & 1 & 8 & 0 & 7 & 6 & 0 & 0 & 32 & 45 & 108 \\
\hline
\end{tabular}

Tabla 3. Lista de especies de mamíferos pequeños terrestres y voladores, $y$ número de individuos registrados en cada uno de los 10 sitios muestreados en San Cristóbal de Las Casas, Chiapas, México. El arreglo taxonómico es el propuesto por Wilson y Reeder (2005).
Los números representa los sitios muestreados: 1. El Cerrito, 2. La Kisst, 3. NaBolom, 4. Saldivar, 5. La Hormiga, 6. La Albarrada, 7. Los Humedales, 8. Navajuelos, 9. La UNICH y 10. El Encuentro.

* Especies introducidas.

Al realizar las comparaciones de riqueza de especies y abundancia relativa de individuos, tanto de murciélagos como de roedores entre cada uno de los espacios verdes (urbanos y no urbanos) muestreados, no se encontraron diferencias significativas $(\mathrm{t}=1.66 ; P \geq 0.5)$.

Análisis de la riqueza y abundancia de roedores entre los espacios verdes urbanos y no urbanos. Para el análisis de la riqueza y abundancia de roedores entre los espacios verdes urbanos y no urbanos muestreados, se agruparon los sitios, tomando en cuenta el tipo de uso de suelo con mayor cobertura y ubicación en la ciudad. El número de especies de roedores entre los diferentes espacios verdes varió, en los urbanos se encontraron tres especies y en los no urbanos cuatro. Sin embargo, las especies de roedores capturadas en los espacios verdes no urbanos, fueron roedores nativos, mientras que en los espacios verdes urbanos la única especie de roedor nativa fue $R$. mexicanus. 
La abundancia entre los dos tipos de espacios verdes varió considerablemente, siendo mayor en los espacios verdes no urbanos, debido al éxito de captura de una especie, $P$. levipes con 42 individuos. La especie más abundante (por el número de individuos registrados) en los espacios verdes urbanos fue $R$. mexicanus, además esta especie fue capturada también en los espacios verdes no urbanos (Tabla 4). Sin embargo, al estimar y comparar el índice de diversidad de Shannon-Wiener de los espacios verdes urbanos $\left(\mathrm{H}^{\prime}=0.859\right)$ y los no urbanos $\left(\mathrm{H}^{\prime}=0.70\right)$, no se encontraron diferencias significativas $(\mathrm{t}=0.39, P=0.70)$.

Análisis de la riqueza y abundancia de murciélagos entre los espacios verdes urbanos y no urbanos. El número de especies de murciélagos difirió en una especie entre los dos tipos de espacios verdes, siendo más alto en los espacios verdes urbanos. Los dos grupos de espacios verdes tuvieron una especie en común, A. lituratus. Por otro lado, la abundancia registrada en los dos grupos de espacios verdes no varió mucho, aunque los dos sitios presentaron una especie dominante. La especie más abundante en los espacios verdes urbanos, fue $A$. lituratus con 18 individuos, mientras que en los espacios verdes no urbanos fue $D$. azteca con 17 individuos (Tabla 4). Al estimar y comparar el índice de diversidad de Shannon-Wiener de los espacios verdes urbanos $\left(\mathrm{H}^{\prime}=0.80\right)$ y no urbanos $\left(H^{\prime}=0.40\right)$, no se encontraron diferencias significativas $(t=1.37, P=0.17)$.

\begin{tabular}{|c|c|c|c|}
\hline \multirow{4}{*}{$\begin{array}{l}\text { Tabla 4. Riqueza y } \\
\text { abundancia de roedores } \\
\text { y murciélagos en espacios } \\
\text { verdes urbanos y no } \\
\text { urbanos en San Cristóbal } \\
\text { de Las Casas, Chiapas, } \\
\text { México. }\end{array}$} & \multirow[b]{2}{*}{ Especies de roedores } & \multicolumn{2}{|c|}{ Tipos de espacios verdes } \\
\hline & & Urbano & No urbano \\
\hline & Peromyscus levipes & 0 & 42 \\
\hline & Peromyscus mexicanus & 0 & 8 \\
\hline & Reithrodontomys mexicanus & 7 & 3 \\
\hline & Sigmodon hispidus & 0 & 2 \\
\hline & Mus musculus* & 1 & 0 \\
\hline & Rattus rattus* & 3 & 0 \\
\hline & Total & 11 & 55 \\
\hline & Especies de murciélagos & & \\
\hline & Artibeus lituratus & 17 & 1 \\
\hline & Dermanura azteca & 0 & 17 \\
\hline & Lasiurus intermedius & 1 & 0 \\
\hline & Lasiurus cinereus & 0 & 1 \\
\hline & Eptesicus fuscus & 4 & 0 \\
\hline & Eptesicus brasiliensis & 1 & 0 \\
\hline *Especies introducidas & Total & 23 & 19 \\
\hline
\end{tabular}

Análisis de la riqueza y abundancia para el total de mamíferos capturados en los espacios verdes urbanos y no urbanos. Considerando el total de mamíferos capturados y registrados en los dos grupos de espacios verdes (urbanos y no urbanos), el número de 
especies fue similar $(n=7)$. Se registraron más especies de murciélagos en los espacios verdes urbanos $(n=4)$ que en los no urbanos $(n=3)$. Por otro lado, se registraron más especies de roedores en los espacios verdes no urbanos $(n=4)$, que en los urbanos $(n$ $=3$ ). En cuanto a la abundancia, los espacios verdes no urbanos presentaron mayor número de registros de mamíferos ( $n=74$ individuos), mientras que los espacios verdes urbanos tuvieron 34 registros. Sin embargo, al estimar y comparar el índice de diversidad de Shannon-Wiener para los espacios verdes urbanos $\left(\mathrm{H}^{\prime}=1.44\right)$ y no urbanos $\left(\mathrm{H}^{\prime}=\right.$ 1.24), no se encontraron diferencias significativas $(t=1.03, P=0.30)$. Cabe mencionar que en tres espacios verdes urbanos (sitios 4,7 y 8 ), no se obtuvo algún registro de fauna.

Correlación entre las variables dependientes (riqueza y abundancia de especies) e independientes (tipos de usos de suelo y la riqueza de especies de árboles). Se consideraron la abundancia y riqueza de especies de todos los mamíferos registrados en los espacios verdes muestreados, dado que no fue posible realizar las pruebas de correlación por grupo taxonómico, ya que los valores de abundancia y riqueza para los roedores y murciélagos son muy bajos. La riqueza total de especies de mamíferos no presentó una relación significativa con las variables independientes estudiadas (los diferentes tipos de usos de suelo y riqueza de especies de árboles; Tabla 5). El análisis de correlación lineal de Pearson mostró un grado mayor de relación entre la abundancia de mamíferos y las variables independientes. De tal manera que la abundancia de mamíferos está negativamente correlacionada con las coberturas urbanas, las zonas de pastoreo y con los espacios abiertos. En contraste, la correlación fue positiva con los espacios verdes y la riqueza de árboles. Para la abundancia se observó un mayor grado de correlación entre la cobertura de espacios verdes $(r=0.66)$ y el número de especies de árboles $(r=0.53)$ registrados en los sitios de muestreo. Sin embargo, sólo la correlación entre la abundancia total de mamíferos y los espacios verdes fue estadísticamente significativa $(P<0.05)$, lo que muestra la magnitud de la correlación (Tab. 5).

\begin{tabular}{lllll}
\hline & $r$ & $r^{2}$ & $t$ & $P$ \\
Riqueza & & & & \\
\cline { 1 - 4 } Espacios abiertos & -0.088 & 0.007 & -0.25 & 0.808 \\
Espacios verdes & 0.278 & 0.077 & 0.819 & 0.436 \\
Zonas urbanas & -0.180 & 0.032 & -0.517 & 0.618 \\
Cuerpos de agua & -0.160 & 0.025 & -0.458 & 0.658 \\
Zonas de pastoreo & -0.446 & 0.199 & -1.410 & 0.196 \\
Riqueza de árboles & 0.389 & 0.151 & 1.196 & 0.265 \\
& & & & \\
Abundancia & & & & \\
\hline Espacios abiertos & -0.174 & 0.030 & -0.501 & 0.629 \\
Espacios verdes & 0.659 & 0.426 & 2.430 & $0.040^{*}$ \\
Zonas urbanas & -0.491 & 0.241 & -1.159 & 0.149 \\
Cuerpos de agua & 0.183 & 0.033 & 0.526 & 0.612 \\
Zonas de pastoreo & -0.474 & 0.225 & -1.526 & 0.165 \\
Riqueza de árboles & 0.526 & 0.277 & 1.753 & 0.117 \\
\hline
\end{tabular}

Tabla 5. Valores de correlación lineal de Pearson entre la riqueza y abundancia de roedores y murciélagos y los tipos de usos de suelo y la riqueza de especies de los árboles de cada sitio. 


\section{Discusión}

El número de especies registradas en este estudio en los dos grupos de espacios verdes (urbanos y no urbanos), muestran que estos espacios albergan más del $40 \%$ del total de las especies registradas para el municipio de San Cristóbal de Las Casas (Naranjo y Espinoza 2001). Se refuerza de esta manera la importancia que tienen los espacios verdes en zonas urbanas, al ser reservorios de la diversidad de fauna. Además, las diferencias en la riqueza y abundancia de especies de roedores y murciélagos entre los dos tipos de espacios verdes, señalan que cada estos dos grupos de mamíferos utilizan los espacios verdes de acuerdo a sus requerimientos biológicos, algunas especies pueden estar usando estos espacios como lugar de paso, de descanso, como refugio y de alimentación.

La captura de mayor número de individuos de especies de roedores nativos en los espacios verdes no urbanos, puede deberse a que en estos espacios, que forman parte de un remanente de bosque, hay mayor disponibilidad de recursos, como alimento, refugio y sitios de reproducción. Por otro lado, en los espacios verdes urbanos se registraron dos especies introducidas, el ratón doméstico (Mus musculus) y la rata negra (Rattus rattus), las cuales prefieren hábitats abiertos urbanos que hábitats naturales (Nelson et al. 1986). En estudios donde han analizado la abundancia de roedores en ciudades, han reportado que el ratón doméstico y las ratas presentan mayor registro en espacios urbanos (Castillo et al. 2003), mientras que los roedores silvestres, son mejor representados en espacios verdes rurales, como vegetación de galería (Johnson et al. 2011).

Cabe señalar que en uno de los espacios verdes urbanos (El Cerrito) se registró una especie de roedor nativo, $R$. mexicanus. Lo anterior concuerda con lo reportado por Godbard et al. (2009) que mencionan que los sitios inmersos en una matriz urbana (como el caso de El Cerrito) y que presentan cierto porcentaje cobertura vegetal o elementos arbóreos propios de la región (en este caso 12 especies arbóreas), permiten albergar poblaciones de fauna nativa.

Las especies nativas de roedores registradas en los espacios verdes no urbanos son, en su mayoría, especies que toleran disturbios humanos y que generalmente son capturadas en zonas de transición (ecotonos) y en bosques perturbados, cultivos y áreas abiertas, como Peromyscus mexicanus, Sigmodon hispidus y Reithrodontomys mexicanus (Horváth et al. 2001; Cruz et al. 2010). Sin embargo, el roedor más abundante en los espacios verdes no urbanos fue $P$. levipes, especie que fue capturada en el interior del bosque en el sitio denominado El Encuentro. Generalmente esta especie es registrada en zonas conservadas, donde encuentra disponibilidad de recursos (Horváth et al. 2001).

Para los murciélagos, no se encontraron diferencias significativas en la diversidad de murciélagos entre los espacios verdes urbanos y los no urbanos. Lo anterior puede llevar a la conclusión que los murciélagos pueden utilizar indistintamente tanto los espacios verdes urbanos como los no urbanos. Sin embargo, la composición de especies de murciélagos varió entre los dos tipos de espacios verdes, compartiendo solamente una especie, Artibeus lituratus. En los espacios verdes urbanos se registraron tres especies de murciélagos insectívoros y sólo un frugívoro, mientras que en los espacios verdes no urbanos se capturaron dos especies frugívoras y un insectívoro. El registro de un mayor 
número de especies de murciélagos insectívoros en los espacios verdes urbanos, de acuerdo a algunos autores, se debe básicamente a que algunas especies que pertenecen a este gremio alimentario presentan mayor actividad en zonas urbanas, al encontrar más cantidad o disponibilidad de alimento en dichas zonas (Ávila-Flores y Fenton 2005; Coleman y Barclay 2012).

Artibeus lituratus y D. azteca, pertenecientes a la familia Phyllostomidae, fueron las especies más abundantes. La primera especie fue más abundante en espacios verdes urbanos, debido a que es una especie generalista y tolerante a los disturbios humanos (Zortéa y Chiarello 1994), lo cual le permite ocupar y utilizar una variedad de sitios, entre ellos zonas urbanas, en donde puede disponer de alimento, como frutos, insectos, polen y hojas (Zortéa y Mendes 1993). Por otra parte, D. azteca se encontró mayormente en espacios verdes no urbanos, es una especie que tiende a ser abundante y con una amplia distribución. Esto debido a que es una especie generalista, al utilizar una variedad de hábitats, desde cafetales hasta bosques templados (Davis 1969; Kraker-Castañeda y Pérez-Consuegra 2011). D. azteca se encuentra asociada a bosques de niebla aunque también se ha capturado en bosques de coníferas, selvas perennifolias, cultivos de plátanos y huertos de mango (Wedster y Jones 1982).

Eptesicus fuscus fue otra especie que se capturó en espacios verdes urbanos, aunque su abundancia no fue muy grande (cuatro individuos en total). Por ser una especie estrictamente aérea de la familia Vespertilionidae, su captura en redes de niebla es muy rara (Barclay 1993), se ha reportado comúnmente a través de detectores acústicos en sitios con perturbación humana (como zonas urbanas o espacios abiertos), y en bosques conservados (Everette et al. 2001; Gehrt y Chelsvig 2004; Ávila-Flores y Fenton 2005).

Sin embargo, hay autores que mencionan que esta especie tiene mayor actividad en áreas urbanas (Rodríguez-Aguilar 2013), debido a que puede utilizar una gran variedad de tipos de refugios, como grietas en rocas, cavidades de árboles y especialmente construcciones humanas (Agosta 2002). Por otro lado, hay autores que sugieren que $E$. fuscus es una especie que se restringe a amplias áreas con vegetación en los grandes centros urbanos (Ávila-Flores y Fenton 2005). Los diversos resultados obtenidos en trabajos anteriores y en este estudio, sugieren que $E$. fuscus es una especie generalista y adaptable a una variedad de paisajes, y el bosque es potencialmente importante para esta especie (Gehrt y Chelsvig 2004).

En este estudio se obtuvieron capturas de un individuo de las especies Lasiurus intermedius, L. cinereus y Eptesicus brasiliensis. La primera especie fue capturada en espacios verdes urbanos. Esta especie ha sido registrada con detectores acústicos en zonas urbanas, áreas de agricultura y en el interior del bosque en la región de Los Altos de Chiapas (Rodríguez-Aguilar 2013). Se sabe muy poco sobre esta especie, dado que no es muy fácil registrar las frecuencias de ultrasonido. Por otra parte, L. cinereus fue capturada en un sitio de los espacios verdes no urbanos (El Encuentro), contrario a lo reportado por Gehrt y Chelsvig (2004), quienes registraron que esta especie tiene actividad en zonas urbanas, al igual que Coleman y Barclay (2012), quienes observaron que esta especie puede tener actividad tanto en áreas urbanas como en zonas conservadas. Los datos obtenidos no permiten saber si el registro de esta especie en El Encuentro refleja una dependencia absoluta de zonas poco perturbadas o, alternativamente, si no pudo ser capturada con redes debido a su tipo de vuelo y hábitos alimenticos (insectívoro aéreo; Shump y Shump 1982). 
Nuestros resultados indican que es muy poco el grado de relación que existe entre la riqueza de especies de roedores y murciélagos y las variables independientes (cobertura de los diferentes usos de suelo y riqueza de árboles). A pesar de los valores tan bajos, se puede apreciar un patrón de correlación negativa entre la riqueza de especies de mamíferos con los espacios abiertos, coberturas urbanas y zonas de pastoreo e incluso con los cuerpos de agua.

Por otro lado, al correlacionar las variables dependientes (riqueza de especies y abundancia de roedores y murciélagos), con las variables independientes (tipos de usos de suelo: espacios abiertos, espacios verdes, zonas urbanas, cuerpos de agua y zonas de pastoreo, y la riqueza de árboles), se encontró solamente una correlación positiva con los espacios verdes, espacios abiertos y con la riqueza de árboles. Resultados similares encontraron Gehrt y Chelsvig (2003), en cuyo estudio existen correlaciones positivas entre variables a escala de paisaje como el uso de suelo y variables de microhábitat de murciélagos, número de lámparas y estructuras hechas por el humano. De la misma manera, Rodríguez-Aguilar (2013) encontró una relación positiva entre variables locales (número de lámparas, densidad poblacional, porcentaje de asfalto, densidad de edificios) con la actividad de murciélagos insectívoros aéreos. Sin embargo, a pesar que en este estudio se encontraron correlaciones tanto positivas como negativas entre las variables independientes a escala de paisaje (cobertura de diferentes usos de suelo en un radio de $500 \mathrm{~m}$ ) y la riqueza y abundancia de roedores y murciélagos, la variable local (riqueza de árboles) fue la que en su mayoría presentó mayor grado de relación. Lo anterior concuerda con los estudios de Gehrt y Chelsvig (2003), quienes sugieren que los efectos de las variables locales son más importantes que las variables a escala de paisaje para explicar las diferencias en la actividad de los murciélagos, dado que los quirópteros al tener la capacidad de volar pueden desplazarse a través de la matriz urbana. De esta manera, algunos murciélagos pueden ser capaces de evadir los efectos negativos de la urbanización y explotar exitosamente otros ambientes. Caso contrario puede ocurrir con los roedores, que por su ámbito de distribución y su capacidad de desplazamiento relativamente corto, las variables a escala local pueden afectar sus poblaciones (Contreras y Rosi 1981; Pearson et al. 1987; García-Estrada et al. 2002; Corbalán y Ojeda 2005).

Por otro lado, al analizar los valores de riqueza y abundancia de árboles en los sitios muestreados, se observó que los espacios verdes urbanos (El Cerrito y Na-Bolom) y no urbanos (La UNICH y El Encuentro), presentaron mayor riqueza y abundancia de especies de árboles, y fueron también las que aportaron el mayor número de especies de roedores y murciélagos. Esto debido a que, en estos sitios hay disponibilidad de recursos, tanto alimenticios como de refugio para los individuos.

En el presente trabajo se encontró que los espacios verdes urbanos y no urbanos en el interior y periferia de la ciudad de San Cristóbal de Las Casas, son importantes para la fauna nativa de roedores y murciélagos. Algunos sitios como La UNICH y El Encuentro (espacios verdes no urbanos) son importantes para la fauna silvestre, al albergar la mayoría de las especies de roedores registradas. De esta manera, la protección de áreas verdes en la periferia de un paisaje urbano permite conservar a una gran proporción de la fauna nativa de la región. 
Por otra parte, se encontró que los espacios verdes en el interior de la ciudad (como Na-Bolom, El Cerrito, La Hormiga y La Albarrada), tienen un papel fundamental para la preservación de poblaciones de roedores nativos y murciélagos, ya que son utilizados por éstos como sitios de descanso, de refugio y como fuentes de recursos alimenticios y de agua. Se espera que los resultados de este estudio sean un punto de partida para la gestión de manejo y mantenimiento de las áreas verdes en SCLC, considerando la sustentabilidad ambiental.

Al CONACYT por la beca otorgada para los estudios de maestría otorgada al autor principal. A las autoridades del municipio de la ciudad de San Cristóbal de Las Casas y a las instituciones particulares por el permiso otorgado para laborar en los espacios verdes urbanos y no urbanos. A J. Bolaños, M. C. Ruíz, L. Tlapaya-Romero y a los estudiantes del servicio social del Laboratorio de la Colección Mastozoológica de El Colegio de La Frontera Sur, Unidad San Cristóbal de Las Casas, por su apoyo en campo. Dos revisores anónimos elevaron la calidad de este artículo con sus comentarios.

Agosta, S. J. 2002. Habitat use, diet and roost selection by the Big Brown Bat (Eptesicus Literatura citada fuscus) in North America: a case for conserving an abundant species. Mammal Review 32:179-198.

Alberti, M. 2009. Advances in urban ecology. Integrating humans and ecological process in urban ecosystems. Springer. Seattle, EE.UU.

Átvarez-Solórzano, J. 1959. Catálogo y claves de los roedores mexicanos. Instituto Politécnico Nacional, Escuela Nacional de Ciencias Biológicas. Tesis de Licenciatura. Ciudad de México, México.

Aubry, A. 2008. San Cristóbal de Las Casas. Su historia urbana, demográfica y monumental 1528-1990. Fray Bartolomé de Las Casas, A. C. San Cristóbal de Las Casas, México.

Ávila-Flores, R., y M. B. Fenton. 2005. Use of spatial features by foraging insectivorous bats in a large urban landscape. Journal of Mammalogy 86:1193-1204.

Barclay, R. M. R. 1993. The biology of Prairie bats. Pp. 352-357 en The 3rd prairie conservation and endangered species workshop (Holroyd, G. L., H. L. Dickson, M. Regnier, y H. Smith, eds.). Provincial Museum of Alberta. Brandon, Canada.

Castillo, E., J. Priotto, A. M. Ambrosio, M. C. Provensal, N. Pini, M. A. Morales, A. Steinmann, y J. J. Polop. 2003. Comensal and wild rodents in an urban area of Argentina. International Biodeterioration y Biodegradation 52:135-141.

Coleman, J. L., y R. M. R. Barclay. 2012. Urbanization and the abundance and diversity of prairie bats. Urban Ecosytem 15:87-102.

Comité Estatal de Información Estadística y Geográfica de Chiapas (CEIEG). 2012. Consultado en: http://www.ceieg.chiapas.gob.mx/home/?page_id=9571\&maccion=957

Contreras, J. R., y M. I. Rosı. 1981. Investigaciones sociobiológicas acerca de roedores silvestres de Mendoza. Serie Científica 22:12-17.

Corbalán, V. E., y R. A. OJedA. 2005. Áreas de acción en un ensamble de roedores del desierto del Monte (Mendoza, Argentina). Mastozoología Neotropical 12:145-152. 
Cruz, E. L, C. Lorenzo, O. G. Retana, y E. C. Sántiz. 2010. Interspecific variability in the abundance of small rodents in the highlands of Chiapas, Mexico. Therya 1:129136.

Davis, W. B. 1969. A review of the small fruit bats (genus Artibeus) of Middle America. Part I. Southwestern Naturalist 14:15-19.

Everette, A. L., T. J. O’Shea, L. E. Ellison, L. A. Stone, y J. L. Mccance. 2001. Bat use of a high-plains urban wildlife refuge. Wildlife Society Bulletin 29:967-973.

Fleming, T. H. 1981. Fecundity, fruiting patterns and seed dispersal in Piper amalago (Piperacea), a bats dispersed tropical shrub. Oceología 51:42-46.

Forman, R. T. T. 2008. Urban region. Ecology and planning beyond the city. Cambridge University Press. Cambridge, EE.UU.

Galindo-Jaimes, L., M. Martínez-Icó, M. López-Carmona, A. Camacho-Cruz, N. RamírezMarcial, y J. A. Santiago-lastra. 2008. Humedales de montaña en Chiapas. Biodiversidad, Conservación y Restauración, A. C. y El Colegio de La Frontera Sur. San Cristóbal de Las Casas, México.

García-Estrada, C., M. L. Romero-Almaraz, y C. Sánchez-Hernández. 2002. Comparison of rodent communities in sites with different degrees of disturbance in deciduous forest of southeastern Morelos, Mexico. Acta Zoológica Mexicana (n. s.) 85:153168.

García-García, A. 2005. La cuenca hidrológica de San Cristóbal de las Casas, Chiapas: entre la gestión local y la nacional. Pp. 42-141 en Problemas Socio-económicos y Experiencias Organizativas en las Cuencas de México (Vargas, S., y E. Mollard, eds.). Instituto Mexicano de Tecnología del Agua, Institut de Recherche pour le Développement. Cuernavaca, México.

Gehrt, S., Y J. Chelsvig. 2003. Bat activity in an urban landscape: Patterns at the landscape and microhabitat scale. Ecological Applications 13:939-950.

Gehrt, S., y J. Chelsvig. 2004. Species-specific patterns of bat activity in an urban landscape. Ecological Applications 14:625-635.

Godbard, M. A., A. J. Dougill, y T. G. Benton. 2009. Scaling up from gardens: biodiversity conservation in urban environments. Trends in Ecology and Evolution 25:90-98.

González-García, A., y A. Gómez-Sal. 2008. Private urban greenspaces or "patios" as a key element in the urban ecology of tropical Central America. Human Ecology 36:291-300.

Gorresen, P. M., M. R. Wiluig, y R. E. Strauss. 2005. Multivariate analysis of scaledependent associations between bats and landscape structure. Ecological Application 15:2126-2136.

Hammer, Q., D. A. T. Harper, y P. D. Ryan. 2001. PAST: Paleontological Statistic software package for education and data analysis. Paleontologia Electronica 4:9.

Horváth, A., I. J. March, y J. D. Wolf. 2001. Rodent diversity and land use in Montebello, Chiapas, Mexico. Studies on Neotropical Fauna and Environment 36:169-176.

Instituto Nacional De Estadística y Geografía (INEGI). 2010. Cuéntame. Consultado En: http://cuentame.inegi.org.mx/poblacion/rur_urb.aspx?tema=P.

Instituto Nacional De Estadística y Geografía (INEGI). 2012. México en cifras. Consultado En: http://www3.inegi.org.mx/sistemas/mexicocifras/default.aspx?e=07. 
Johnson, J., R. Lever, y J. Storm. 2011. Small mammal community structure in urban greenways. USC Upstate Undergraduate Research Journal 4:19-22.

Kuingbeil, B. T., y M. R. Wituıg. 2009. Guild-specific responses of bats to landscape composition and configuration in fragmented Amazonian rainforest. Journal of Applied Ecology 46:1-11.

Kraker-Castañeda, C., y G. Pérez-Consuegra. 2011. Contribución de los cafetales bajo sombra en la conservación de murciélagos en la Antigua Guatemala, Guatemala. Acta Zoológica Mexicana (n. s.) 27:291-303.

Kunz, T. H. 1996. Methods of marking bats. Pp. 304-310 in Measuring and monitoring biological diversity standard methods for mammals (Wilson, R. Cole, N. Rudran, y Foster, eds.) Smithsonian Press. Washington, EE.UU.

Loram, A., P. Warren, K. Thompson, y K. Gaston. 2011. Urban domestic gardens: the effects of human interventions on garden composition. Environmental Management 48:808-824.

Mahan, C. G., Y T. J. O'Connell. 2005. Small mammal use of suburban and urban parks in central Pennsylvania. Northeastern Naturalist 12:307-314.

Mason, J., C. Moorman, G. Hess, y K. Sinclair. 2007. Designing suburban greenways to provide habitat for forest-breeding birds. Landscape and Urban Planning 80:153164.

McDonnell, M. J., y S. T. A. Pickett. 1990. Ecosystem structure and function along urbanrural gradients: an unexploited opportunity for ecology. Ecology 71:1232-1237.

Mcdonnell, M. J., y S. T. A. Pickett. 1993. Humans as components of eco- systems: The ecology of subtle human effects and populated areas. Springer-Verlag. New York, EE.UU.

McKinneY, M. L. 2002. Urbanization, biodiversity, and conservation. BioScience 52:883-90.

McKinney, M. L. 2008. Effects of urbanization on species richness: A review of plants and animals. Urban Ecosystem 11:161-176.

Medellín, R. A., H. Arita, y O. Sánchez. 1997. Identificación de los murciélagos de México, clave de campo. Publicaciones Especiales, Asociación Mexicana de Mastozoología, A. C. Ciudad de México, México.

Mera-Ovando, L. M. 1984. Regionalización de la Subregión San Cristóbal de las Casas, Chiapas. Tesis de Licenciatura en Biología. Universidad Nacional Autónoma de México, Facultad de Ciencias. Ciudad de México, México.

Miller, J. R., Y R. J. Новвs. 2002. Conservation where people live and work. Conservation Biology 16:330-337.

Naranjo, E., y E. Espinoza. 2001. Los mamíferos de la reserva ecológica Huitepec, Chiapas, México. Revista Mexicana de Mastozoología 5:58-67.

Nelson, B. C., M. B. Madon, y A. Tilzer. 1986. The complexities at the interface among domestic wild rodents, fleas, pets and man in urban plague ecology in Los Angeles, country, California. Vertebrate Pest Conference Proceedings Collection 1:88-96.

Pearson, O. P., S. Martin, y J. Bella. 1987. Demography and reproduction of the silky desert mouse (Eligmodontia) in Argentina. Fieldiana Zoology 23:433-446.

Ramírez-Marcial, N., A. Camacho-Cruz, M. Martínez-Icó, A. Luna-Gómez, D. Golicher, y M. González-Espinosa. 2010. Árboles y arbustos de los bosques de montaña en Chiapas. El Colegio de La Frontera Sur. San Cristóbal de Las Casas, México. 
ReID, A. F. 1997. A field guide to the mammals of central and southeast Mexico. Oxford University Press. Nueva York, EE.UU.

Rodríguez-Aguilar, G. 2013. Efecto de la urbanización sobre el ensamble de murciélagos insectívoros aéreos en Los Altos de Chiapas, México. Tesis de Maestría. El Colegio de la Frontera Sur. San Cristóbal de Las Casas, México.

Romero-Almaraz, M. L., C. Sánchez-Hernández, C. García-Estrada, y R. D. Owen. 2007. Mamíferos pequeños. Manual de técnicas de captura, preparación, preservación y estudio. Universidad Nacional Autónoma de México. Ciudad de México, México.

Sauvajot, R. M., M. Buechner, y C. M. Schonewald. 1998. Patterns of human disturbance and response by small mammals and birds in chaparral near urban development. Urban Ecosystems 2:279-297.

Shump, K. A. JR., y A. U. Shump. 1982. Lasiurus cinereus. Mammalian Species 185:1-5.

Smith, R. M., K. J. Gaston, P. H. Warren, y K. Thompson. 2006. Urban domestic gardens (IX): composition and richness of the vascular plant flora, and implications for native biodiversity. Biological Conservation 129:312-322.

SuKopp, H., y P. Werner. 1982. Nature in cities: A report and review of studies and experiments concerning ecology, wildlife and nature conservation in urban and suburban areas. Strasbourg (France): Council of Europe. Nature and Environment Series 28:48-94.

VÁsQuez, L. 2010. La agricultura urbana como elemento promotor de la sustentabilidad urbana. Situación actual y potencial en San Cristóbal de las Casas, Chiapas. Tesis de Maestría. El Colegio de la Frontera Sur. San Cristóbal de Las Casas, México.

Vitousek, P. M., H. A. Mooney, J. Lubchenco, y J. M. Melillo. 1997. Human domination of the Earth's ecosystems. Science 277:494-499.

Wedster, WM. D., y J. K. Jones, JR. 1982. Artibeus aztecus. Mammalian Species 177:1-3.

Wilson, D. E., y D. M. Reeder (eds.). 2005. Mammal Species of the World, a Taxonomic and Geographic Reference, tercera edición. Johns Hopkins Press. Baltimore, EE.UU.

Wu, J. 2008. Toward a landscape ecology of cities: beyond buildings, trees, and urban forests. Pp. 10-28 en Ecology, planning, and management of urban forests international perspectives (Carreriro, M. M., Y. C. Song, y J. G. Wu, eds.). Springer. Nueva York, EE.UU.

Zortéa, M., y A. G. Chiarello. 1994. Observations on the big fruit-eating bat, Artibeus lituratus in an urban reserve of south-east Brazil. Mammalia 58:665-670.

Zortéa, M., y S. L. Mendes. 1993. Folivory in the Big Fruit-eating Bat, Artibeus lituratus (Chiroptera: Phyllostomidae) in eastern Brazil. Journal of Tropical Ecology 9:117120.

\author{
Sometido: 30 de mayo de 2014 \\ Revisado: 26 de junio de 2014 \\ Aceptado: 13 de agosto de 2014 \\ Editor asociado: Cristina Macswiney \\ Diseño gráfico editorial: Gerardo Hernández
}


